\title{
Quasi-Cherenkov Radiation from Relativistic Particles Passing Through a Photonic Crystal
}

\author{
V.G. Baryshevsky*, A.A. Gurinovich ${ }^{\dagger}$
}

July 22, 2021

\begin{abstract}
Research Institute for Nuclear Problems, Belarusian State University, Bobruiskaya 11, 220030 Minsk, Belarus
\end{abstract}

\begin{abstract}
The expressions for spectral-angular distribution of quasi-Cherenkov radiation emitted by a relativistic particle traversing a photonic crystal are derived. It is shown that for a relativistic particle, passing through a photonic crystal formed by periodically strained threads, the intensity of quasi-Cherenkov radiation emitted at small angles to the direction of particle motion, as contrasted to ordinary Cherenkov radiation, exhibits anisotropic properties as the the photon momentum is rotated about the direction of particle motion (as the crystal is rotated about the direction of particle motion at fixed-angle observation of the outcoming photon).

The intensity of quasi-Cherenkov radiation in terahertz and optical ranges is shown to be high enough to allow the experimental study of quasi-Cherenkov radiation in these frequency ranges.
\end{abstract}

\section{Introduction}

Diffraction radiation of photons from relativistic particles moving in crystals (natural or artificial spatially periodic structures) has come under intensive theoretical and experimental investigation in recent years.

\footnotetext{
*E-mail:bar@inp.bsu.by

${ }^{\dagger}$ E-mail:gur@inp.bsu.by
} 
The ever-growing interest to the study of radiation and emission processes in spatially periodic structures was inspired by pioneer research into microwave oscillators using radiation from an electron beam in a periodic slowwave structure (traveling wave tube, backward wave oscillator, etc.) [1,2].

In 1953, Smith and Purcell [3] made the next step and observed generation of incoherent radiation at visible wavelengths by using a finely-focused electron beam propagating close to the surface of a metal diffraction grating (at the distance $\delta \leq \frac{\lambda \beta \gamma}{4 \pi}, \delta$ is the beam impact parameter, $\lambda$ is the radiation wavelength, $\beta=v / c, v$ is the electron beam velocity, and $\gamma$ is the electron Lorentz factor).

The Smith-Purcell effect belongs to a general class of diffraction radiation effects induced by the interaction of electrons with a medium. Diffraction of waves associated with the electromagnetic field of the electron by an obstacle leads to the so-called diffraction radiation [4].

The feature of diffraction radiation is that the radiation frequency of photons emitted forward in the direction of particle motion increases as the particle energy is increased, passing from optical to X-ray and $\gamma$ ranges [5]. (In X-ray and $\gamma$ ranges, this type of radiation is called the resonant radiation.) In 1971, it was shown [6] that for particles moving in a crystal, the induced Vavilov-Cherenkov effect arises in the X-ray range, and thus the spontaneous effect arises too [7 9]. The feature of this effect is that the $\mathrm{X}$-ray-quantum frequency is independent of the particle energy even for radiation in the forward direction. Later this radiation was called the parametric (quasi-Cherenkov) X-ray radiation (PXR) and observed experimentally in 1985 [7, 8, 10, 11]. This effect can arise because the Bragg diffraction of X-ray photons in crystals has a result that even in the X-ray range, where the refractive index of a medium $n(\omega)<1$, there are waves for which the refractive index $n(\omega)$ can be greater than unity. A similar phenomenon also occurs in artificial three(two)-dimensional periodic structures, now frequently referred to as photonic (electromagnetic) crystals [12]. The "grid" structure formed by periodically strained dielectric threads was experimentally studied in [13, where it was shown that "grid" photonic crystals can have a sufficiently high $Q$ factor $\left(10^{4}-10^{8}\right)$. Induced radiation from an electron beam in a photonic crystal formed by periodically strained metallic threads is described in [12]. Radiation generators using two- or three-dimensional periodic structures (natural, photonic, electromagnetic crystals) are called volume free electron lasers (VFEL).

A significant number of theoretical and experimental studies of parametric (quasi-Cherenkov) X-ray radiation are presently available [7, 8, 14].

Behind a theoretical description of parametric X-ray radiation is the application of the dynamical theory of diffraction of X-ray radiation, developed in works on Bragg diffraction of X-ray photons [15-19]. The backbone that underlies the dynamical theory of diffraction is the possibility to use loworder perturbation theory to describe scattering of X-ray quanta by atoms.

Typically, in optical and microwave ranges, the perturbation theory does not apply to describe photon scattering by scatterers that form an electromagnetic (photonic) crystal as this theory does in the X-ray range. Nevertheless, as we have demonstrated earlier [12], using the scattering amplitude to describe the interaction of photons with a scattering center we can derive equations defining dynamical diffraction in electromagnetic crystals, and so 
we can use many of the results obtained in the PXR theory [12, 20, 21 to describe the emission of photons from relativistic particles moving in electromagnetic (photonic) crystals.

This paper derives the expressions for spectral-angular distribution of quasi-Cherenkov radiation emitted by a relativistic particle traversing a photonic crystal. It is shown that when a relativistic particle moves in a photonic crystal formed by periodically strained threads, the intensity of Cherenkov radiation emitted at small angles to the direction of particle motion, as contrasted to ordinary Cherenkov radiation, exhibits anisotropic properties as the photon momentum is rotated about the direction of particle motion (as the crystal is rotated about the direction of particle motion at fixed-angle observation of the outcoming photon).

It is shown that the intensity of quasi-Cherenkov radiation in terahertz and optical ranges is high enough to allow the experimental study of quasiCherenkov radiation.

\section{Spectral-angular distribution of radiation pro- duced by a particle passing through a pho- tonic crystal}

To describe the process of photon emission by a particle traversing matter, we shall use the method developed in [7,8].

Knowing the field $\vec{E}(\vec{r}, \omega)$, produced by a particle at a large distance from the target, we can find both the spectral-angular density of radiant energy per unit solid angle, $W_{\vec{n} \omega}$, and the differential number of emitted photons, $d N_{\vec{n} \omega}=\frac{W_{\vec{n} \omega}}{\hbar \omega}$,

$$
W_{\vec{n} \omega}=\frac{e r^{2}}{4 \pi^{2}} \overline{|\vec{E}(\vec{r}, \omega)|^{2}}
$$

The vinculum here means averaging over all possible states of the radiating system.

To find the field $\vec{E}(\vec{r}, \omega)$, we need to solve Maxwell's equations describing the interaction of particles with a medium. The transverse solution can be found using the Green function of these equations, which satisfies the expression [7, 8, 22]:

$$
G=G_{0}+G_{0} \frac{\omega^{2}}{4 \pi c^{2}}(\hat{\varepsilon}-1) G .
$$

Here $G_{0}$ is the transverse Green function of Maxwell's equation at $\hat{\varepsilon}=1$ (it is given, for example, in [23]) and $\hat{\varepsilon}$ is the permittivity tensor of the medium.

Using $G$, we can find the field we are concerned with

$$
E_{n}(\vec{r}, \omega)=\int G_{n l}\left(\vec{r}, \vec{r}^{\prime}, \omega\right) \frac{i \omega}{c^{2}} j_{0 l}(\vec{r}, \omega) d^{3} r^{\prime}
$$

where $n, l=x, y, z$ and $j_{0 l}(\vec{r}, \omega)$ is the Fourier transformation of the $l-t h$ component of the current produced by moving charged particles. In the linear approximation, the current is determined by the particle's velocity and trajectory, which can be found by solving the equation of particle motion. Under quantum-mechanical consideration, the current $j_{0}$ is the current of transition of the particle-medium system from one state to another. 
According to [7, 8], the Green's function at $r \rightarrow \infty$ is expressed through the solution of homogeneous Maxwell's equations $E_{\vec{k} l}^{(-) s}(\vec{r}, \omega)$ containing at infinity a converging spherical wave:

$$
\lim _{r \rightarrow \infty} G_{n l}\left(\vec{r}, \vec{r}^{\prime}, \omega\right)=\frac{e^{i k r}}{r} \sum_{s} e_{n}^{s} E_{\vec{k} l}^{(-) s *}\left(\vec{r}^{\prime}, \omega\right),
$$

where $\vec{k}=k \frac{\vec{r}}{r}, \vec{e}^{s}$ is the unit polarization vector, $s=1,2$, and $\vec{e}^{1} \perp \vec{e}^{2} \perp \vec{k}$.

The solution $E_{\overrightarrow{k l}}^{(-) s *}\left(\vec{r}^{\prime}\right)$ for large distances from the area occupied by the medium has the form

$$
\vec{E}_{k}^{(-) s}\left(\vec{r}^{\prime}, \omega\right)=\vec{e}^{s} e^{i \vec{k} \vec{r}^{\prime}}+\mathrm{const} \frac{e^{-i k r^{\prime}}}{r^{\prime}} .
$$

The solution $\vec{E}_{\vec{k}}^{(-) s}(\vec{r}, \omega)$ is associated with the solution of homogeneous Maxwell's equations $\vec{E}_{\vec{k}}^{(+) s}(\vec{r}, \omega)$ that describes a plane-wave scattering by a crystal and contains at infinity a diverging spherical wave $\vec{E}_{k}^{(+) s}(\vec{r}, \omega)=$ $\vec{e}^{s} e^{i \vec{k} \vec{r}}+$ const $\frac{e^{i k r}}{r}$, namely, (see [7, 8]):

$$
\vec{E}_{\vec{k}}^{(-) s *}=\vec{E}_{-\vec{k}}^{(+) s}
$$

Using (3) and (4), we obtain

$$
\vec{E}_{n}(\vec{r}, \omega)=\frac{e^{i k r}}{r} \frac{i \omega}{c^{2}} \sum_{S} \vec{e}_{n}^{s} \int \vec{E}_{\vec{k}}^{(-) s *}(\vec{r}, \omega) \vec{j}_{0}\left(\vec{r}^{\prime}, \omega\right) d^{3} r^{\prime}
$$

As a result, the spectral density of radiant energy for photons with polarization $s$ can be written in the form:

$$
\begin{gathered}
W_{\vec{n}, \omega}^{s}=\frac{\omega^{2}}{4 \pi^{2} c^{2}} \overline{\left|\int \vec{E}_{\vec{k}}^{(-) s *}(\vec{r}, \omega) \vec{j}_{0}(\vec{r}, \omega) d^{3} r\right|^{2}}, \\
\vec{j}_{0}(\vec{r}, \omega)=\int e^{i \omega t} \vec{j}_{0}(\vec{r}, \omega) d t=e Q \int e^{i \omega t} \vec{v}(t) \delta(\vec{r}-\vec{r}(t)) d t,
\end{gathered}
$$

where $e Q$ is the charge of the particle, $\vec{v}(t)$ and $\vec{r}(t)$ are the velocity and the trajectory of the particle at time $t$.

Substitution of (7) into (6) gives

$$
d N_{\vec{n}, \omega}^{s}=\frac{e^{2} Q^{2} \omega}{4 \pi^{2} \hbar c^{3}} \overline{\left|\int \vec{E}_{\vec{k}}^{(-) s *}(\vec{r}(t), \omega) \vec{v}(t) e^{i \omega t}\right|^{2}} d t .
$$

Integration in (8) is performed over the entire domain of particle motion. Solving homogeneous Maxwell's equations instead of inhomogeneous significantly simplifies the analysis of the radiation problem and enables considering different cases of radiation with due account of multiple scattering.

We shall further concern ourselves with the generation of parametric quasi-Cherenkov radiation by a relativistic charged particle moving at constant velocity $(\vec{v}=$ const) through a photonic crystal, i.e. [see (44)],

$$
d N_{\vec{n}, \omega}^{s}=\frac{e^{2} Q^{2} \omega}{4 \pi^{2} \hbar c^{3}} \overline{\left|\int \vec{E}_{-\vec{k}}^{(+) s}(\vec{r}(t), \omega) \vec{v}(t) e^{i \omega t}\right|^{2}} d t .
$$


According to (8), to find the number of photons emitted by a particle traversing a crystal plate, we need to find the explicit expressions for the solutions $\vec{E}_{\vec{k}}^{(-) s}$. To do this, we only need to know the solutions $\vec{E}_{\vec{k}}^{(+) s}$ describing refraction and diffraction of photons in the crystal, since

$$
\vec{E}_{\vec{k}}^{(-) s}=\left(\vec{E}_{-\vec{k}}^{(+) s}\right)^{*},
$$

as we have stated earlier in this section.

\section{Refraction and diffraction of electromagnetic waves in photonic crystals formed by period- ically strained threads}

Refraction and diffraction of waves in natural and artificial (photonic, electromagnetic) crystals have been intensively studied since the first publications on the dynamical theory of X-ray diffraction in crystals appeared [15, 24]. The feature of the dynamical theory of X-ray diffraction in crystals is that it allows the application of the perturbation theory to describe scattering of X-ray quanta by atoms [25,26].

Further analysis has shown that even when the perturbation theory does not apply to describe scattering by a single center belonging to a crystal (as, e.g., in the case of neutrons or slow electrons), the methods similar to those used in the dynamical theory of X-ray diffraction work well to describe diffraction of radiation in crystals.

According to [26], the direction of scattered waves (formed through diffraction in crystals) leaving the plane-parallel plate is uniquely determined by the incident direction and the momentum (energy, wavelength) of the incident radiation. Moreover, it is determined in the same way as in the elementary kinematic Laue theory of interference developed for thin plates, for which the effects of refraction may be neglected: the projections onto the crystal surface of the wave vectors of scattered and incident waves differ by the projection of the reciprocal lattice vector, $\vec{\tau}_{\perp}$, onto the crystal surface. The possible refraction only leads to the intensity redistribution among the scattered (diffracted) waves.

According to [8, 12, 27], the dynamical theory of diffraction enables developing a common approach to the description of photon emission from relativistic particles in natural and artificial crystals.

Taking this approach to the analysis of the emission process, we may use similar formulas, varying only the form of the coefficients that define frequency-angular and time properties of the produced radiation. It should be stated here that in contrast to the Laue-Ewald dynamical theory, the perturbation theory does not, as a rule, apply to describe scattering of electromagnetic waves by an elementary scatterer (crystal cell) in a photonic crystal.

The theory of electromagnetic-wave propagation in photonic crystals has been actively studied, see, e.g., [28 37]. The authors focused on calculating the energy-band structure for such frequency ranges and parameters of photonic crystals for which a photon forbidden zone exists (the analogue of the electron forbidden zone in crystals, i.e., the region of total Bragg reflection). 
The authors of [38 40] gave a detailed analysis of the band structure and the field inside crystals for the case of two-dimensional crystals formed by periodically strained metallic threads.

At the same time, as has been shown earlier in section 2, to investigate the emission of waves by particles traversing a photonic (electromagnetic) crystal, we need to know the expression for the electromagnetic field both inside and outside the crystal. This means that we need to know the solutions to Maxwell's equations that describe refraction and diffraction of waves in a photonic crystal.

In this connection we generalized the dynamical theory of diffraction in crystals to the case of photonic (electromagnetic) crystals [12. Since under this approach the major parameter is the amplitude of wave scattering by a scatterer, the approach becomes applicable to the description of refraction and diffraction in crystals built from the elements (threads, spheres) with arbitrary dielectric permittivity, in which case the perturbation theory does not apply to describe scattering by a center.

Because the theory [12] will further be used to describe quasi-Cherenkov radiation in photonic crystals, let us recall the main aspects of the theory of refraction and diffraction of electromagnetic waves in the case of interest - two-dimensional periodic electromagnetic crystals built from periodically strained threads [12].

Let us suppose that a plane electromagnetic wave characterized by the electric field vector $\vec{E}(\vec{r})=\vec{e} e^{i \vec{k} \vec{r}}$ is incident on the thread. Here $\vec{e}$ is the unit polarization vector, $\vec{k}$ is the wave vector, and $\vec{r}$ is the radius-vector. Let us also suppose that the wave is incident perpendicular to the thread's axis, i.e., along the $z$-axis. The thread is placed at the origin of coordinates and its axis coincides with the $x$-axis (see Fig. 1).

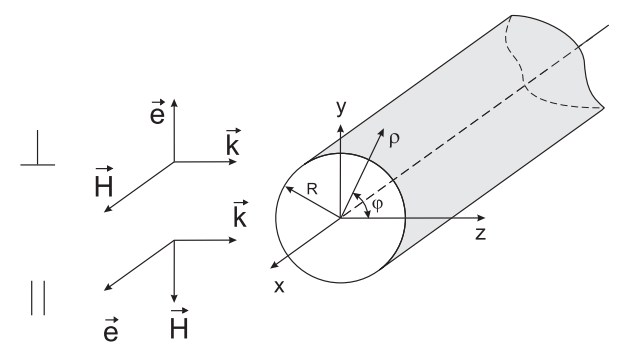

Figure 1: Coordinates and vectors

Let us assume that the thread is a cylinder of radius $R$. In this case, the problem of wave scattering by a cylinder has a well-known solution (see, e.g., 41]).

In particular, if the polarization vector $\vec{e}$ is parallel to the thread's axis $\left(\vec{e}=\vec{e}^{\|}\right)$, then outside the region occupied by the thread, i.e., at $\rho>R$, the expression for the wave formed through scattering can be written as a superposition of the incident $\vec{E}_{0}$ and scattered $\vec{E}_{s c}$ waves:

$$
\begin{gathered}
\vec{E}=\vec{e} \Psi(\rho, z) \\
\Psi(\rho, z)=e^{i k z}+\sum_{n=-\infty}^{\infty} i^{n} a_{n}^{\|} H_{n}^{(1)}(k \rho),
\end{gathered}
$$


where $\rho=(y, z)$ is the transverse coordinate, $H_{n}^{(1)}$ is the Hankel function of order $n$, and $k=\frac{\omega}{c}$ is the wave number.

If the dielectric permittivity and magnetic permeability of the thread are $\varepsilon(\omega)$ and $\mu(\omega)$, respectively, then the expressions for the amplitudes $a_{n}$ can be written in the form

$$
a_{n}^{\|}=\frac{-J_{n}\left(k_{t} R\right) J_{n}^{\prime}(k R)+\sqrt{\frac{\varepsilon}{\mu}} J_{n}^{\prime}\left(k_{t} R\right) J_{n}(k R)}{J_{n}\left(k_{t} R\right) H_{n}^{(1) \prime}(k R)-\sqrt{\frac{\varepsilon}{\mu}} J_{n}^{\prime}\left(k_{t} R\right) H_{n}^{(1)}(k R)},
$$

where $J_{n}$ are the Bessel functions of order $n, k_{t}=k \sqrt{\varepsilon(\omega) \mu(\omega)}$; for nonmagnetic metals $\mu(\omega)=1$ and $\varepsilon(\omega)=1+i \frac{4 \pi \sigma_{t}}{\omega}$, where $\sigma_{t}$ is the conductivity of the thread's material.

Let us now consider scattering by a thread of a wave whose polarization is orthogonal to the thread's axis, $\vec{e} \perp 0 x$. It is convenient in this case to consider rescattering of waves using the amplitude of scattering of a magnetic component of the electromagnetic wave [42], because the wave's magnetic field vector $\vec{H}_{0}=\vec{e} \| e^{i k z}$ is parallel to the thread's axis. For the scattered wave, the magnetic field strength at $\rho>R$ has the form

$$
\vec{H}=\vec{e}_{\|} \Psi_{H}(\rho, z), \Psi_{H}(\rho, z)=e^{i k z}+\sum_{n=-\infty}^{\infty} i^{n} a_{n}^{\perp} H_{n}^{(1)}(k \rho) e^{i n \varphi}
$$

where $\varphi$ is the azimuth angle (see Fig. 1).

$$
a_{n}^{\perp}=\frac{-J_{n}\left(k_{t} R\right) J_{n}^{\prime}(k R)+\sqrt{\frac{\mu}{\varepsilon}} J_{n}^{\prime}\left(k_{t} R\right) J_{n}(k R)}{J_{n}\left(k_{t} R\right) H_{n}^{(1) \prime}(k R)-\sqrt{\frac{\mu}{\varepsilon}} J_{n}^{\prime}\left(k_{t} R\right) H_{n}^{(1)}(k R)},
$$

Let us suppose that the radiation wavelength $\lambda$ is much greater than the thread's radius $R$. In this case, analyzing scattering of a wave whose electric vector $\vec{E}_{0}$ has a polarization parallel to the thread's axis, we can retain in the sum (11) only the term proportional to $a_{0}^{\|}[12$. Moreover, for well-conducting threads, the amplitude $a_{0}^{\|} \gg a_{n}^{\perp}$ [12]. As a consequence, the wave whose polarization is parallel to the thread is scattered more strongly than the wave whose vector $\vec{E}_{0}$ is polarized orthogonally to the thread [10,11]. Let us also note that according to the analysis [42, if the wave's electric field vector $\vec{E}_{0}$ is orthogonal to the thread's axis, then the following relationships hold true for all $|n|>1: a_{0}^{\perp} \approx a_{ \pm 1}^{\perp}, a_{0}^{\perp} \approx a_{0}^{\perp}$, and $a_{ \pm 1}^{\perp} \gg a_{n}^{\perp}$. This implies that for a wave with orthogonal polarization, three terms will retain in the sum (13), as contrasted to scattering of a wave whose electric field vector $\vec{E}_{0}$ has a polarization parallel to the thread's axis.

\subsection{Refraction of waves by a set of scatterers}

Let an electromagnetic wave be incident on a set of threads with coordinates $\rho_{l}=\left(y_{l}, z_{l}\right)$.

For the sake of concreteness, we shall first consider a wave with polarization parallel to the threads: $\vec{e}=\vec{e}\|\| 0 x$. The scattered wave can be represented as a superposition of waves scattered by single threads

$$
\Psi=e^{i k z}+a_{0}^{\|} \sum_{l} H_{0}^{(1)}\left(k\left|\vec{\rho}-\vec{\rho}_{l}\right|\right) e^{i k z_{l}} .
$$


Using the integral representation of the Hankel function $H_{0}^{(1)}(k \rho)$, we can express the scattered wave as

$$
\Psi=e^{i k z}+A_{0}^{\|} \sum_{l} \int_{-\infty}^{\infty} \frac{e^{i k \sqrt{\left|\vec{\rho}-\vec{\rho}_{l}\right|^{2}-x^{2}}}}{\sqrt{\left|\vec{\rho}-\vec{\rho}_{l}\right|^{2}-x^{2}}} d x e^{i k z_{l}}
$$

where $A_{0}^{\|}=-\frac{i}{\pi} a_{0}^{\|}$and $\left|\vec{\rho}-\vec{\rho}_{l}\right|^{2}=\left(y-y_{l}\right)^{2}+\left(z-z_{l}\right)^{2}$.

Let us consider a wave passing through a layer formed by the threads whose axes lie in the plane $x 0 y$ at a distance $d_{y}$ from one another. Let us suppose that the transverse dimension $L_{\perp}$ of the layer is much greater than the distance $d_{y}$ and the radiation wavelength $\left(L_{\perp} \gg d_{y}\right.$ and $\left.L_{\perp} \gg \lambda\right)$.

Summation over the coordinates $y_{l}$ in this case gives the following expression for $\Psi$ :

$$
\Psi=e^{i k z}+\frac{2 \pi i A_{0}^{\|}}{k d_{y}} e^{i k z}=\left(1+\frac{2 \pi i A_{0}^{\|}}{k d_{y}}\right) e^{i k z} .
$$

This expression reflects a well-known fact that a plane wave of unit amplitude, scattered by a plane layer of scatterers, converts into a plane wave of complex amplitude $1+\frac{2 \pi i A_{0}^{\|}}{k d_{y}}$. Let us suppose that the parameter $\left|\frac{2 \pi i A_{0}^{\|}}{k d_{y}}\right| \ll 1$. In this case, the expression for the wave multiply scattered by $m$ number of planes spaced from one another at a distance $d_{z}$ can be presented [12] in the from $\Psi=e^{i k n_{\|} z}$, where $n_{\|}$is the refractive index that can be expressed as:

$$
n_{\|}=1+\frac{2 \pi}{d_{y} d_{z} k^{2}} A_{0}^{\|}
$$

It follows from (18) that in this case, the real part of the refractive in$\operatorname{dex} \operatorname{Re} n_{\|}=n_{\|}^{\prime}=1+\frac{2 \pi}{d_{y} d_{z} k^{2}} \operatorname{Re} A_{0}^{\|}$, while its imaginary part $\operatorname{Im} n_{\|}=n_{\|}^{\prime \prime}=$ $\frac{2 \pi}{d_{y} d_{z} k^{2}} \operatorname{Im} A_{0}^{\|}$.

The expressions of the form (18) for the refractive index can also be derived for a medium made up of wires chaotically distributed in the plane $(z 0 y)$. In this case, the quantity $\frac{1}{d_{y} d_{z}}$ is replaced by $\rho_{y z}$, the density of scatterers in the plane $(z 0 y)\left(\frac{1}{d_{y}} \longrightarrow \rho_{y}\right.$, the density of threads along the $y$-axis, and $\frac{1}{d_{z}} \longrightarrow \rho_{z}$, the density of threads along the $z$-axis).

If the threads are chaotically distributed along the $y$-axis, then $\rho_{y z}=\rho_{y} \frac{1}{d_{z}}$, where $\rho_{y}$ is the density of threads along the $y$-axis. When the threads are distributed chaotically along the $z$-axis and periodically along the $y$-axis, the density $\rho_{y z}=\rho_{z} \frac{1}{d_{y}}$, where $\rho_{z}$ is the density of threads along the $z$-axis.

Thus for a medium built from chaotically distributed threads, we have

$$
n_{\|}=1+\frac{2 \pi \rho_{y z}}{k^{2}} A_{0}^{\|}
$$

Let us recall here that $A_{0}^{\|}=-\frac{i}{\pi} a_{0}^{\|}$.

A wave with polarization orthogonal to the thread's axis $(\vec{e} \perp 0 x)$ can be considered along similar lines.

The expression for the refractive index $n_{\perp}$ is similar to (19) with $A_{0}^{\|}$ replaced by $A_{0}^{\perp}=-\frac{i}{\pi}\left(a_{0}^{\perp}+2 a_{1}^{\perp}\right)$. As a result, we have

$$
n_{\perp}=1+\frac{2 \pi \rho_{y z}}{k^{2}}\left(-\frac{i}{\pi} a_{0}^{\perp}-\frac{2 i}{\pi} a_{1}^{\perp}\right)
$$


It is noteworthy that the imaginary part of the refractive index (18) and (19) is nonzero when the wave is scattered by a perfectly conducting thread when absorption in the thread is absent. The result (19), describing the wave passage through a medium formed by chaotically distributed scatterers, is valid because the waves scattered at nonzero angles have random phases, since the threads are distributed chaotically, and the scattered waves do not interfere. As a result, they lead to attenuation of the initial wave.

In the case (18), the imaginary part must be missing, because in a crystalline medium scattering of waves is nonrandom. The attenuation of waves in crystals is determined by their absorption in the threads [12] (see Sec. 3.2).

As the radiation frequency increases and the wavelength becomes comparable with the radius of the thread $(k R \geq 1)$, other terms in (11) and (13) also make contributions to scattering of waves by a thread. To find the refractive index in this case, we can also perform averaging over the location(s) of the threads in the plane transverse with respect to the $z$-axis, but this procedure involves cumbersome calculations: first you need to express the Hankel functions of an arbitrary index $n$ in terms of $H_{0}$, and then the problem is reduced to the problem considered earlier in this section.

Interestingly, in the case when the parameter $k d_{z} \geq 1$, i.e., the radiation wavelength is less than the inter-thread distance along the $z$-axis, the refractive index can be found in a more simple way. To do this, we make use of the asymptotic representation of the Hankel function for $k \rho \gg 1$

$$
H_{n}^{(1)}(k \rho) \approx \sqrt{\frac{2}{\pi k \rho}} e^{i\left(k \rho-\frac{\pi}{2}\left(n+\frac{1}{2}\right)\right)}=(-i)^{n} H_{0}^{(1)}(k \rho)
$$

As a consequence, when the distance from the thread is much greater than the wavelength $\left(\rho \gg \frac{1}{k}\right)$, we obtain the following expression for the scattered wave having the polarization $\vec{e}=\vec{e}_{\|} \| 0 x$

$$
\begin{gathered}
\vec{E}=\vec{e}_{\|} F^{\|}(\varphi) \sqrt{\frac{2}{\pi k \rho}} e^{i k \rho} e^{-i \frac{\pi}{4}}=\vec{e} F^{\|}(\varphi) H_{0}^{(1)}(k \rho) \\
F^{\|}(\varphi)=\sum_{n=-\infty}^{\infty} a_{n}^{\|} e^{i n \varphi} .
\end{gathered}
$$

Similar expressions can be written for a wave having the electric vector $\vec{e}=$ $\vec{e}_{\perp} \perp 0 x$ (the magnetic field is directed along $0 x$ ).

$$
\begin{gathered}
\vec{H}=\vec{e}_{\perp} \sum_{n=-\infty}^{\infty} i^{n} a_{n}^{\perp} H_{n}(k \rho) F^{\perp}(\varphi) \sqrt{\frac{2}{\pi k \rho}} e^{i k \rho} e^{-i \frac{\pi}{4}}=\vec{e} F^{\perp}(\varphi) H_{0}^{(1)}(k \rho) \\
F^{\perp}(\varphi)=\sum_{n=-\infty}^{\infty} a_{n}^{\perp} e^{i n \varphi}
\end{gathered}
$$

Let us consider a wave passing through the layer of $N$ number of scatterers in the plane $z=z_{l}$. The electric field strength at point $z$ is described by the expression:

$$
\vec{E}(\vec{r})=\vec{e} \sum_{l=1}^{N} \sum_{n=-\infty}^{\infty}\left(a_{n}^{\|} e^{\left(i n \varphi_{l}\right)} H_{0}^{(1)}\left(k\left|\vec{\rho}-\overrightarrow{\rho_{l}}\right|\right)\right) e^{i k z_{l}}
$$




$$
\left|\vec{\rho}-\vec{\rho}_{l}\right|^{2}=\left(y-y_{l}\right)^{2}+\left(z-z_{l}\right)^{2},
$$

where $\varphi_{l}$ is the azimuth angle between $\vec{k}$ and the direction $\left(\vec{\rho}-\overrightarrow{\rho_{l}}\right)$.

Upon summation over the positions of the threads in the plane $x 0 y$, using the integration representation of the Hankel functions

$$
H_{0}^{(1)}\left(k\left|\vec{\rho}-\overrightarrow{\rho_{l}}\right|\right)=-\frac{i}{\pi} \int_{-\infty}^{\infty} \frac{e^{i k \sqrt{\left(\vec{\rho}-\overrightarrow{\rho_{l}}\right)^{2}+x^{2}}}}{\sqrt{\left(\vec{\rho}-\overrightarrow{\rho_{l}}\right)^{2}+x^{2}}} d x
$$

and the relations

$$
\cos \varphi_{l}=\frac{z-z_{l}}{\left(y-y_{l}\right)^{2}+\left(z-z_{l}\right)^{2}}, \sin \varphi_{l}=\frac{y-y_{l}}{\left(y-y_{l}\right)^{2}+\left(z-z_{l}\right)^{2}},
$$

we obtain the integral expression, which, through integration by parts, can be reduced to the form $\left(z>z_{l}\right)$ :

$$
\Psi=e^{i k z}+\frac{2 \pi i}{k y} A^{\|}(0)+\text { the terms } \sim \frac{1}{k\left(z-z_{l}\right)},
$$

where

$$
A^{\|}(0)=-\frac{i}{\pi} F^{\|}(0)=\left(-\frac{i}{\pi}\right) \sum_{n=-\infty}^{\infty} a_{n}^{\|}
$$

Summation of the waves that have traversed several planes gives the expression for the refractive index, similar to (19) with $A_{0}^{\|}$replaced by $A^{\|}(0)$

$$
n_{\|}=1+\frac{2 \pi \rho_{y z}}{k^{2}} A^{\|}(0) \text {. }
$$

Similarly, for a wave with polarization $\vec{e}_{\perp}$, the summation of waves (24) gives the refractive index of the form (32) with $A^{\|}(0)$ replaced by $A^{\perp}(0)$ :

$$
n_{\perp}=1+\frac{2 \pi \rho_{y z}}{k^{2}} A^{\perp}(0)
$$

where $A^{\perp}(0)=-\frac{i}{\pi} F^{\perp}(0)=\left(-\frac{i}{\pi}\right) \sum_{m} a_{m}^{\perp}$.

The wave propagating through 'matter' built from metallic threads can be written in the form

$$
\vec{E}=E_{0 \|} \vec{e}_{\|} e^{i k n_{\|} z}+E_{0 \perp} \vec{e}_{\perp} e^{i k n_{\perp} z} .
$$

Since $n_{\|} \neq n_{\perp}$, such 'matter' is birefringent [10,11], i.e., as the wave propagates, its linear polarization converts into circular and so on. Because $\operatorname{Im} n_{\|} \neq \operatorname{Im} n_{\perp}$, such 'matter' exhibits dichroism, and the law of absorption has a conventional form for the polarization $e_{\|}\left(e_{\perp}\right)$

$$
\left|E_{\|(\perp)}\right|^{2}=\left|E_{0 \|(\perp)}\right|^{2} e^{-2 k n_{\|(\perp)} z}
$$

On the other hand, upon introducing the total scattering cross section by a thread $\left(\sigma_{\|(\perp)}\right)$, we can write the law of absorption in the form

$$
\left|E_{\|(\perp)}\right|^{2}=\left|E_{0 \|(\perp)}\right|^{2} e^{-\rho_{e z} \sigma_{\|(\perp)} z} .
$$

From this follows that

$$
\sigma_{\|(\perp)}=\frac{4 \pi}{k} \operatorname{Im} A_{\|(\perp)}(0)=-\frac{4}{k} \operatorname{Re} F_{\|(\perp)}(0)=\frac{4}{k} \sum_{n}\left|a_{n_{\perp(\|)}}\right|^{2} .
$$


Let us note that the cross section dimension in a $2 \mathrm{D}$ case is not a squared length as in a $3 \mathrm{D}$ case, but a length. The amplitude $A(0)$ is a non-dimensional quantity.

Equation (37) can also be derived from (22). In a two-dimensional case, the amplitude $f$ of scattering at the angle $\varphi$ is defined as the factor of a cylindrical wave, $\frac{e^{i k \rho}}{\sqrt{\rho}}: f(\varphi)=\sqrt{\frac{2}{\pi k}} e^{-i \frac{\pi}{4}} F(\varphi)$. Let us note that this amplitude has the dimension $\sqrt{[l]}$, the square root of the length. The amplitude $f(\varphi)$ allows finding the differential scattering cross section that is defined by the ratio of the energy flux density in the wave scattered at the angle $\varphi$ to that in the incident wave

$$
\frac{d \sigma}{d \varphi}=|f(\varphi)|^{2}=\frac{2}{\pi k}|F(\varphi)|^{2}
$$

From this we immediately obtain:

$$
\sigma=\int \frac{d \sigma}{d \varphi} d \varphi=\frac{4}{k} \sum_{n}\left|a_{n}\right|^{2}
$$

Now let us consider a wave reflected in a backward direction from the layer of cylinders distributed in the plane $z=z_{l}$.

In this case, (26)-(29) yield the following expression for the wave reflected from the plane and having the electric field vector parallel to the $x$-axis:

$$
\begin{gathered}
E_{r e f}^{\|}\left(z<z_{l}\right)=\frac{2 \pi i \rho_{y}}{k} A^{\|}(\pi) e^{2 i k z_{l}} e^{-i k z} \\
A^{\|}(\pi)=-\frac{i}{\pi} F^{\|}(\pi)=-\frac{i}{\pi} \sum_{n=-\infty}^{\infty}(-1)^{n} a_{n}^{\|}
\end{gathered}
$$

Then we have the following expression for a wave reflected from $N$ number of planes:

$$
E_{r e f}^{\|}(z)=\frac{2 \pi i \rho_{y}}{k} A^{\|}(\pi) \sum_{l=1}^{N} e^{2 i k z_{l}} e^{-i k z}
$$

When the layer thickness is much greater than the wavelength, we can perform integration instead of summation and thus obtain the following expression for $E_{r e f}^{\|}$

$$
E_{r e f}^{\|}(z)=-\frac{\pi \rho_{y z}}{k^{2}} A^{\|}(\pi) e^{-i k z}
$$

We can see that, as expected, the amplitude of the wave reflected from the layer is determined by the amplitude $F(\pi)$ of the wave scattered in the direction of reflection [see (22) and (41)], i.e., backwards $\left(F^{\|}(\pi)\right.$ - for a wave with parallel polarization and $F^{\perp}(\pi)$ - for a wave with orthogonal polarization).

At the same time, the refractive index is determined by the amplitude $F^{\|}(0)$ of a wave scattered in the forward direction [see (31) and (32)]. From this it immediately follows that when scattering is non-isotropic (versus isotropic), the mirror-reflection coefficient cannot be found from the refractive index (dielectric permittivity) [8]. 


\subsection{Refraction and diffraction in a photonic crystal}

Now let us consider how the expression for the refractive index changes as the wave moves in the crystal.

According to [12], when performing summation of scattered waves in the case of electromagnetic crystals, it is essential that rescattering of waves be taken into account. This leads to vanishing of the imaginary part of the refractive index for crystals formed by perfectly conducting metallic threads (with chaotically distributed threads, the imaginary part of the refractive index is nonzero, as shown earlier).

It is also demonstrated in [12] that when electromagnetic crystals are built from periodically strained parallel metallic threads, taking rescattering into account allows us to obtain the following expression for the refractive index of a wave whose electric field vector $\vec{E}_{0}$ is parallel to the thread's axis (when the radiation wavelength is much greater than the thread's radius $\lambda>>R$ ):

$$
n_{\|}^{2}=1+\frac{4 \pi}{\Omega_{2} k^{2}} \frac{A_{0}}{1+i \pi A_{0}-2 C A_{0}}=1+\frac{\eta^{\|}}{k^{2}},
$$

where

$$
\eta^{\|}=\frac{4 \pi}{\Omega_{2}} \frac{A_{0}^{\|}}{1+i \pi A_{0}^{\|}-2 C A_{0}^{\|}},
$$

$\Omega_{2}$ is the volume of the unit cell of the $2 \mathrm{D}$-crystal, $C=0.5722$ is the Euler constant, and $A_{0}^{\|}=-\frac{i a_{0}^{\|}}{\pi}$.

Let us note that when $k R \ll 1$, the term $\left(2 C A_{0}^{\|}\right)$is small as compared to unity, and so it can be discarded.

Let us now consider the refractive index $n_{\perp}$ of a wave whose electromagnetic field vector is orthogonal to the thread's axis, $\vec{E}_{0} \perp 0 x$. As shown in [12], at $\lambda \gg R$, the inequality $\left|n_{\perp}^{2}-1\right| \ll\left|n_{\|}^{2}-1\right|$ holds for well-conducting threads. The explicit expressions for the refractive index $n_{\perp}$ were derived in [42] [see formulas (49) and (54) below].

Consequently, for a photonic crystal of thickness $L$ satisfying the condition $\frac{1}{k\left|n_{\|}-1\right|} \leq L<\frac{1}{k\left|n_{\perp}-1\right|}$, refraction can be neglected if the wave's polarization is $\vec{E}_{0} \perp 0 x$, but should be considered if its polarization is $\vec{E}_{0} \| 0 x$

Note here that according to [42, at $k R \ll 1$ the refractive index $n_{\perp}$ for well-conducting metallic threads is greater than unity $\left(n_{\perp}>1\right)$, so at the lengths $L \geq \frac{1}{k\left|n_{\perp}-1\right|}$, the refraction of waves with polarization $\vec{E}_{0} \perp 0 x$ becomes important, and we can observe the Vavilov-Cherenkov effect [42].

Now let us consider the diffraction of waves in electromagnetic crystals. The theory of wave diffraction by periodically strained threads in the case when the wavelength is much greater than the thread's radius $(k R \ll 1)$ is given in [12]. According to [12], the effective dielectric permittivity of a crystal for a wave with $\vec{E}_{0} \| 0 x$ can be written as

$$
\varepsilon^{\|}(\vec{r})=\sum_{\vec{\tau}} \varepsilon_{\tau}^{\|} e^{i \vec{\tau} \vec{r}}
$$

where the Fourier expansion coefficients $\varepsilon_{\tau}^{\|}$of the function with crystalline periodicity $\varepsilon^{\|}(\vec{r})$ have the form

$$
\varepsilon_{0}^{\|}=n_{\|}^{2}=1+\chi_{\|}, \chi_{\|}=\frac{\eta_{\|}}{k^{2}}=\frac{c^{2} \eta_{\|}}{\omega^{2}}, \varepsilon_{\tau}^{\|}=\varepsilon_{-\tau}^{\|}=\chi_{\|}
$$


where $\chi_{\|}$is the polarizability, $\vec{\tau}$ is the reciprocal lattice vector of a $2 \mathrm{D}$ periodic photonic crystal, and $\vec{\tau}=\left(0, \tau_{y}, \tau_{z}\right)$.

Note that according to the analysis [12], $\eta_{\|}<0$ for high-conductivity metallic threads.

As a result, the quantities $\varepsilon_{\tau}^{\|}$in this case are similar to the quantities $\varepsilon_{\tau}$ describing the diffraction of hard X-rays. Recall that in the X-ray range, $\varepsilon_{\tau}<0$ for frequencies much greater than characteristic atomic frequencies, $\varepsilon_{0}=n^{2}=1-\frac{\omega_{L}^{2}}{\omega^{2}}<1$, where $\omega_{L}$ is the plasma frequency [16, 17, 19].

The results obtained here allow us to apply the elaborate dynamical theory of X-ray diffraction to the description of wave diffraction and photon emission by relativistic particles in photonic crystals.

Let us note that the equations that allow describing diffraction in electromagnetic crystals in the case of anisotropic scattering of waves with polarization parallel $\vec{E} \| 0 x$ and orthogonal $\vec{E} \perp 0 x$ to the thread were derived in [42].

\section{Quasi-Cherenkov radiation from charged par- ticles in photonic crystals built from period- ically strained threads}

Let a relativistic charged particle of charge $e Q(|e|$ is the electron charge, $Q$ is the integer) pass through a photonic crystal. According to the analysis in Section 3.2, in the case of well-conductive threads, the refractive index for a wave whose polarization $\vec{E}$ is parallel to the threads is $n_{\|}<1$. This means that a particle uniformly moving in the crystals will not emit photons with such polarization because of the Vavilov-Cherenkov effect.

However, the situation becomes entirely different when considering that during the emission the photons produced in the crystal can undergo diffraction.

According to [8], the diffraction of virtual photons in crystals can be described with a set of refractive indices $n_{\mu}(\omega, k)$, some of which may appear greater than unity even if the refractive index $n$ in the absence of diffraction was less than unity. Particularly, when a diffracted wave is formed in a crystal through Bragg diffraction, along with a wave with wave vector $\vec{k}$ (two-wave Laue-Bragg diffraction), the interaction of the electromagnetic wave with the crystal is characterized by two refractive indices: $n_{1}(\omega, k)>1$ and $n_{2}(\omega, k)<1$.

As a consequence, two types of waves propagating in a photonic crystal correspond to each polarization: a fast wave $\left(n_{2}<1\right)$ and a slow one $\left(n_{1}>1\right)$. The conditions for Cherenkov radiation can be fulfilled for a slow wave, but not for a fast one.

A fast wave is emitted at the vacuum-crystal boundary in a similar manner as in the case of ordinary transition radiation.

Let a relativistic particle move in a photonic crystal at constant velocity $\vec{v}$. It follows from (8) and (9) that spectral-angular distribution of the number of photons emitted by a particle moving through the crystal is determined 
by the expression of the form:

$$
\frac{d^{2} N^{s}}{d \Omega d \omega}=\frac{e^{2} Q^{2} \omega}{4 \pi^{2} \hbar c^{3}}\left|\int \vec{E}_{\vec{k}}^{s(-) *}(\vec{r}(t), t) \vec{v} e^{i \omega t} d t\right|^{2}
$$

where $\vec{k}=\frac{\omega}{c} \vec{n}, \vec{n}$ is the unit vector directed to the point of observation, and $\vec{E}_{\vec{k}}^{s(-) *}=\vec{E}_{-\vec{k}}^{s(+)}$.

In view of (46), to obtain the spectral-angular distribution, we need to find the solutions that determine the plane-wave diffraction in a photonic crystal. This solution was found in [12 for a wave with electric field vector parallel to the thread's axis $\vec{E} \| 0 x$ for $k R \ll 1$, in which case wave scattering by a thread is isotropic. In the general case of anisotropic scattering, the equations describing the dynamical diffraction of waves with polarizations parallel $\vec{E} \| 0 x$ and orthogonal $\vec{E} \perp 0 x$ to the thread's axis in photonic crystals were derived in 42

We shall make use of the results reported in [12,42] to find the spectralangular distribution of quasi-Cherenkov radiation in the case when two coupled waves are produced through diffraction in a photonic crystal (two-wave diffraction case). The waves propagating in a crystal are described by the following set of equations:

$$
\begin{aligned}
& \left(\frac{k^{2} c^{2}}{\omega^{2}}-1-g_{0}^{s *}\right) \vec{E}_{\vec{k}}^{s(-)}-g_{-\vec{\tau}}^{s *} \vec{E}_{\vec{k}_{\tau}}^{s(-)}=0 \\
& \left(\frac{k^{2} c^{2}}{\omega^{2}}-1-g_{0}^{s *}\right) \vec{E}_{\vec{k}_{\tau}}^{s(-)}-g_{\vec{\tau}}^{s *} \vec{E}_{\vec{k}}^{s(-)}=0
\end{aligned}
$$

where $\vec{k}_{\tau}=\vec{k}+\vec{\tau}, \vec{\tau}$ is the reciprocal lattice vector, and $s=1,2$ (1 and 2 correspond to the polarizations $\vec{E}$ parallel and orthogonal to the threads, respectively).

$$
\begin{gathered}
g_{0}^{(1)} \equiv g_{0}^{\|}=\frac{4 \pi c^{2}}{\omega^{2} \Omega_{2}} \frac{A_{0}^{\|}}{1+i \pi A_{0}^{\|}}, g_{\tau}^{(1)} \equiv g_{\tau}^{\|}=g_{0}^{\|}, \\
g_{0}^{(2)} \equiv g_{0}^{\perp}=\frac{4 \pi c^{2}}{\omega^{2} \Omega_{2}}\left[\frac{A_{0}^{\perp}}{1+i \pi A_{0}^{\perp}}+\frac{A_{1}^{\perp}}{1+i \frac{\pi}{2} A_{1}^{\perp}}\right], \\
g_{\vec{\tau}}^{(2)} \equiv g_{\vec{\tau}}^{\perp}=\frac{4 \pi c^{2}}{\omega^{2} \Omega_{2}}\left[\frac{A_{0}^{\perp}}{1+i \pi A_{0}^{\perp}}+\frac{A_{1}^{\perp}}{1+i \frac{\pi}{2} A_{1}^{\perp}} \cos (\vec{k}, \vec{k}+\vec{\tau})\right],
\end{gathered}
$$

Because the diffraction process in a periodic medium has a general character, equations (47) are similar to the equations of the dynamical theory of diffraction of X-rays and neutrons in crystals. Of course, for X-rays or neutrons, the coefficients similar to $g_{\tau}$, appearing in (47), will be different.

Particularly, given the threads for which $|\varepsilon-1|,|\mu-1| \gg 1$, the angular dependence of the coefficient $g_{\tau}^{(2)}=g_{\tau}^{\perp}$, describing scattering of a wave whose electric-field polarization vector lies in the diffraction plane $(\vec{k}, \vec{k}+\vec{\tau})$, is defined by two terms: independent $\left(\sim A_{0}^{\perp}\right)$ and dependent $\left(\sim A_{1}^{\perp}\right)$ of the scattering angle. For X-rays, the angular dependence of the quantity, analogous to $g_{\tau}^{\perp}$, is determined alone by the term proportional to $\cos (\vec{k}, \vec{k}+\tau)$. If the inequality $|\varepsilon-1|,|\mu-1| \ll 1$ for dielectric (magnetic) permeability of the thread holds, then the terms proportional to $A_{0}^{\perp}$ in the expression for $g_{\tau}^{\perp}$ can 
be neglected (the coefficient $A_{0}^{\perp} \ll A_{1}^{\perp}[42]$ ), and the angular dependence $g_{\tau}^{\perp}$ for the thread will appear similar to the quantity $g_{\tau}^{\perp}$ for X-rays.

Let us note here that when a photonic crystal is made in a homogeneous isotropic crystal (e.g. when the threads are submerged in a substance whose dielectric permittivity $\varepsilon_{a v}=1+\chi_{a v}$ ), the quantity $\chi_{a v}$ must appear in (48) and (49). For the case when $\chi_{a v} \sim 1$ and $g_{01} g_{\tau} \ll \chi_{a v}$, the diffraction of waves in a periodic medium was considered in 43 .

The requirement that the solution to linear system (46) exist yields the dispersion equation defining vectors $\vec{k}$ that may be present in the crystal. These vectors are convenient to write in the form (compare with [22]).

$$
\vec{k}_{\mu s}=\vec{k}+\vec{\kappa}_{\mu s}^{*} \vec{N}, \quad \kappa_{\mu s}^{*}=\frac{\omega}{c \gamma_{0}} \varepsilon_{\mu s}^{*},
$$

where $\mu=1,2$ and $\vec{N}$ is the unit vector normal to the crystal surface through which the particle enters

$$
\varepsilon_{\mu s}=\frac{1}{4}\left[\left(1+\beta_{1}\right) g_{0}^{s}-\beta_{1} \alpha_{B}\right] \pm \frac{1}{4}\left\{\left[\left(1-\beta_{1}\right) g_{0}^{s}+\beta_{1} \alpha_{B}\right]^{2}+4 \beta_{1} g_{\vec{\tau}}^{s} g_{-\tau}^{s}\right\}^{-1 / 2},
$$

$\alpha_{B}=\left(2 \vec{k} \vec{\tau}+\tau^{2}\right) k^{-2}$ is detuning from the Bragg condition $\left(\alpha_{B}=0\right.$ if the Bragg condition is exactly fulfilled)

$$
\gamma_{0}=\vec{n}_{\gamma} \cdot \vec{N}, \quad \vec{n}_{\gamma}=\frac{\vec{k}}{k}, \quad \beta_{1}=\frac{\gamma_{0}}{\gamma_{1}}, \quad \gamma_{1}=\vec{n}_{\gamma \tau} \cdot \vec{N}, \quad \vec{n}_{\gamma \tau}=\frac{\vec{k}+\vec{\tau}}{|\vec{k}+\vec{\tau}|} .
$$

The general solution of (46) inside the crystal reads

$$
\vec{E}_{\vec{k}}^{(-) s}(\vec{r})=\sum_{\mu=1}^{2}\left[\vec{e}^{s} \Phi_{\vec{k}_{\mu}}^{s} \exp \left(i \vec{k}_{\mu s} \vec{r}\right)+\vec{e}_{\tau}^{s} \Phi_{\vec{k}_{\mu \vec{\tau}}}^{s} \exp \left(i \vec{k}_{\mu s \tau} \vec{r}\right)\right]
$$

The unit vector $\vec{e}^{(1)}=\vec{e}_{\tau}^{(1)}$ lies in the plane formed by the thread's axis (x-axis) and the $0 z$-axis that is orthogonal to the thread; we assume that the angle between $\vec{e}_{\tau}^{(1)}$ and the $0 x$-axis is small. As the angle between $\vec{e}^{(1)}$ and the $0 x$-axis increases, the solutions come in a more complicated form, and so we shall not consider this case here. Vectors $\vec{e}^{(2)}$ and $\vec{e}_{\tau}^{(2)}$ are orthogonal to the thread's axis and lie in the plane formed by vectors $\vec{k}$ and $\vec{k}+\vec{\tau} ; \vec{e}_{\tau}^{(2)} \perp \vec{k}+\vec{\tau}$.

Joining this solution with the solutions to Maxwell's equations in a vacuum, we can find the explicit form of the field $\vec{E}_{\vec{k}}^{(-) s}(\vec{r})$ throughout the area.

Thus, in the case of two-wave dynamical diffraction, a photonic crystal is characterized by two effective refractive indices for each polarization:

$$
n_{\mu s}=1+\varepsilon_{\mu s}, \mu=1,2
$$

Let us recall here that in the case under consideration $\left|\varepsilon_{\mu s}\right| \ll 1$.

When the parameter $\alpha_{B}$ increases and becomes much larger than $g_{\tau}$, the amplitude of the diffracted wave diminishes rapidly, and only the initial wave remains whose refractive index is

$$
n_{s}=1+\frac{1}{2} g_{0}^{s}
$$

Analyzing the radiation process in crystals, we should distinguish between two cases: quasi-Cherenkov radiation during the diffraction of the emitted photon in Bragg and Laue geometries. 
(a) Let us consider quasi-Cherenkov radiation in the Laue geometry. In the Laue geometry, the incident (transmitted) and diffracted waves leave the crystal through the same surface: $k_{z}>0, k_{z}+\tau_{z}>0$, where the $z$-axis is parallel to the normal $N$ to the crystal surface and directed towards the interior of the crystal. Joining the solutions to Maxwell's equations on the crystal surface via (46), (51), and (52), we can obtain for the Laue case:

$$
\begin{aligned}
& \vec{E}_{\vec{k}}^{(-) s}=\left\{\vec{e}^{-}\left[-\sum_{\mu=1}^{2} \xi_{\mu s}^{0 *} e^{-i \frac{\omega}{\gamma_{0}} \varepsilon_{\mu s}^{*} L}\right] e^{i \vec{k} \vec{r}}+e_{\vec{\tau}}^{s} \beta_{1}\left[\sum_{\mu=1}^{2} \xi_{\mu s}^{\tau *} e^{-i \frac{\omega}{\gamma_{0}} \varepsilon_{\mu s}^{*} L}\right] e^{i \vec{k}_{\tau} \vec{r}}\right\} \theta(-z) \\
& +\left\{\vec{e}^{-s}\left[-\sum_{\mu=1}^{2} \xi_{\mu s}^{0 *} e^{-i \frac{\omega}{\gamma_{0}} \varepsilon_{\mu s}^{*}(L-z)}\right] e^{i \vec{k} \vec{r}}+e_{\vec{\tau}}^{s} \beta_{1}\left[\sum_{\mu=1}^{2} \xi_{\mu s}^{\tau *} e^{-i \frac{\omega}{\gamma_{0}} \varepsilon_{\mu s}^{*}(L-z)}\right] e^{i \vec{k}_{\tau} \vec{r}}\right\} \\
& \times \theta(L-z) \theta(z)+\vec{e}^{-} e^{i \vec{k} \vec{r}} \theta(z-L),
\end{aligned}
$$

where

$$
\begin{gathered}
\xi_{1,2 s}^{0}=\mp \frac{2 \varepsilon_{2,1 s}-g_{0}^{s}}{2\left(\varepsilon_{2 s}-\varepsilon_{1 s}\right)} ; \\
\xi_{1,2 s}^{\tau}=\mp \frac{g_{-\tau}^{s}}{2\left(\varepsilon_{2 s}-\varepsilon_{1 s}\right)} ; \\
\theta(z)= \begin{cases}1, & \text { if } z \geq 0 \\
0, & \text { if } z<0 .\end{cases}
\end{gathered}
$$

Equation (55) coincides with a similar expression derived in [22] and goes over to that in [22] if the quantities $\varepsilon_{\mu s}, g_{0}$, and $g_{\tau}$ defined by (48), (49), (50), and (51) are replaced by $\varepsilon_{\mu s}, g_{0}$, and $g_{\tau}$ defined for X-ray diffraction (see [22]).

Upon substituting (55) into (8), we can find the differential number of quasi-Cherenkov-radiation quanta having the polarization $\vec{e}_{s}$ and emitted in the forward direction at small angles to the particle velocity direction in the Laue geometry

$$
\begin{aligned}
& \frac{d^{2} N_{0 s}^{L}}{d \omega d \Omega}=\frac{e^{2} Q^{2} \omega}{4 \pi^{2} \hbar c^{3}}\left(e^{s} \vec{v}\right)^{2} \mid \sum_{\mu=1,2} \xi_{\mu s}^{0} e^{i \frac{\omega}{c \gamma_{0}} \varepsilon_{\mu s} L}\left[\frac{1}{\omega-\vec{k} \vec{v}}-\frac{1}{\omega-\vec{k}_{\mu s}^{*} \vec{v}}\right] \\
& \times\left.\left[e^{i\left(\omega-\vec{k}_{\mu s}^{*} \vec{v}\right) T}-1\right]\right|^{2},
\end{aligned}
$$

where $T=L / c \gamma_{0}$ is the particle's time of flight, $\vec{e}_{1} \|[\vec{k} \vec{\tau}]$ and $\vec{e}_{2} \|\left[\vec{k} \vec{e}_{1}\right]$.

It is obvious that (56) is very similar to the expression for the spectralangular distribution of Cherenkov and transition radiations in a medium having the refractive index $n_{\mu s}=k_{z \mu s} / k_{z}=1+\kappa_{\mu s} / k_{z}=1+\varepsilon_{\mu s}$.

Spectral-angular distribution of photons in the diffraction direction $\vec{k}_{\tau}=$ $\vec{k}+\vec{\tau}$ can be derived from (56) by substituting

$$
\begin{aligned}
& \vec{e}_{s} \rightarrow \vec{e}_{s \tau}, \quad \xi_{\mu s}^{0} \rightarrow \beta_{1} \xi_{\mu s}^{\tau}, \\
& \xi_{1(2) s}^{\tau}= \pm \frac{g_{\tau}^{s}}{2\left(\varepsilon_{1 s}-\varepsilon_{2 s}\right)} \\
& \vec{k} \rightarrow \vec{k}_{\tau}, \quad \vec{k}_{\mu s} \rightarrow \vec{k}_{\tau \mu s}=\vec{k}_{\mu s}+\tau .
\end{aligned}
$$

(b) Now let us consider quasi-Cherenkov radiation in a photonic crystal in the Bragg geometry. In this case, the diffracted wave leaves the crystal through the same surface on which the incident wave falls. 
Joining the solution to Maxwell's equations (52) with the solution to Maxwell's equations in a vacuum, we can obtain the expressions describing the Bragg geometry.

Spectral-angular distribution of photons emitted in the direction of particle motion can be derived by substituting (compare with [22]) $\xi_{\mu s}^{0} \rightarrow \gamma_{\mu s}$

$$
\gamma_{1(2) s}^{0}=\frac{2 \varepsilon_{2(1) s}-g_{0}^{s}}{\left(2 \varepsilon_{2(1) s}-g_{0}^{s}\right)-\left(2 \varepsilon_{1(2) s}-g_{0}^{s}\right) e^{i \frac{\omega}{\gamma_{0}}\left(\varepsilon_{2(1) s}-\varepsilon_{1(2) s}\right) L}}
$$

Spectral-angular distribution of photons emitted in the diffraction direction can be obtained from (56) by substituting

$$
\vec{e}_{s} \rightarrow \vec{e}_{s \tau}, \quad \vec{k} \rightarrow \vec{k}_{\tau}, \quad k_{\mu s} \rightarrow \vec{k}_{\mu \tau s}, \quad \xi_{\mu s}^{0} e^{i \frac{\omega}{\gamma_{0}} \varepsilon_{\mu s} L} \rightarrow \gamma_{\mu s}^{\tau},
$$

where

$$
\gamma_{1(2) s}^{\tau}=-\frac{\beta_{1} g_{\tau}^{s}}{\left(2 \varepsilon_{2(1) s}-g_{0}^{s}\right)-\left(2 \varepsilon_{1(2) s}-g_{0}^{s}\right) e^{i \frac{\omega}{\gamma_{0}}\left(\varepsilon_{2(1) s}-\varepsilon_{1(2) s}\right) L}} .
$$

The formulas derived here describe quasi-Cherenkov radiation in photonic crystals formed by periodically strained threads, as well as transition radiation and Cherenkov radiation that is possible for a state with polarization orthogonal to the threads far from the region of diffraction reflection.

Let us analyze the expressions derived here.

We shall now consider quasi-Cherenkov radiation in a photonic (electromagnetic) crystal built from high-conductivity metallic threads under the conditions when $k R=\frac{2 \pi R}{\lambda} \ll 1$ ( $\lambda$ is the radiation wavelength). In this case, $\varepsilon_{1,2}^{\perp} \ll \varepsilon_{1,2}^{\|}$, as stated earlier. Because the number of quanta emitted at the diffraction peak is proportional to $\xi_{\mu s}^{\tau} \sim g_{\tau}^{s}$, the number of quasiCherenkov radiation quanta $N_{\perp}$ with polarization orthogonal to the thread's axis is much less than the number of quasi-Cherenkov radiation quanta $N_{\|}$ with electric-field-vector polarization in the plane formed by the thread's axis and the wave vector $\vec{k}$ of the emitted wave.

As a result, the polarization of the emitted photon lies mainly in the plane formed by the thread's axis and the photon's wave vector.

Let now a particle move along the $z$-axis, in the direction orthogonal to the entrance surface of the crystal.

We shall focus on the angular distribution of quasi-Cherenkov radiation emitted either in the forward direction at small angles with respect to particle velocity or in the backward direction at small angles with respect to the direction $(-\vec{v})$ (backward Bragg reflection). In this case, the radiation intensity of photons whose polarization $\vec{e} \| 0 x$ equals zero if the photon wave vector $\vec{k}$ lies in the plane $(y, z)$, and only a significantly less intense radiation remains, whose polarization is $\vec{e} \perp 0 x$.

If we rotate vector $\vec{k}$ about $\vec{v}$ (about the z-axis), for vector $\vec{k}$ lying in the $(x, z)$ plane, the radiation intensity of photons with polarization $\vec{e}$ in the $(x, z)$ plane attains its maximum. As is seen, the radiation intensity exhibits axial asymmetry.

This absence of the azimuth symmetry in the angular distribution of quasi-Cherenkov radiation in the considered photonic crystal is in sharp contrast to the case of ordinary Cherenkov radiation or parametric X-ray radiation emitted in the forward direction, of which the azimuth symmetry of angular distribution with respect to particle velocity is typical. 
Let us estimate the number of quasi-Cherenkov quanta emitted by a particle after it has passed through the crystal of thickness $L$.

It follows from (56) (for detail see [7,44]) that the number of the emitted quanta can be approximately estimated by a simple formula

$$
N_{p h} \approx \frac{\alpha Q^{2}\left|g_{\tau}^{\|}\left(\omega_{B}\right)\right|^{2} \frac{\omega_{B}}{c} L}{\sin ^{2} \theta_{B}}
$$

i.e., in the case of interest, when $N^{\|}$, we have

$$
N_{p h}^{\|} \approx \frac{\alpha Q^{2}}{\sin ^{2} \theta_{B}}\left|\frac{4 \pi c^{2}}{\omega_{B}^{2} \Omega_{2}} \frac{A_{0}^{\|}}{1+i \pi A_{0}^{\|}}\right|^{2} k_{B} L
$$

where $\alpha$ is the fine structure constant.

Recall here that the Bragg diffraction conditions can be written as

$$
m \lambda_{B}=2 d \sin \theta_{B},
$$

where $d$ is the lattice spacing. Thus, we can readily obtain for $\omega_{B}$ and $k_{B}$

$$
\omega_{B}=m \frac{\pi c}{d \sin \theta_{B}}=\frac{\tau c}{2 \sin \theta_{B}}, k_{B}=\frac{\tau}{2 \sin \theta_{B}}
$$

where $\tau=\frac{2 \pi}{d} m$ is the reciprocal lattice vector. form:

Using the expression for the Bragg frequency, we can recast $N_{p h}^{\|}$in the

$$
N_{p h}^{\|} \approx \alpha Q^{2} \frac{1}{4 \sin ^{2} \theta_{B}} \frac{1}{k_{B}^{4} \Omega_{2}^{2}}\left|4 \pi \frac{A_{0}^{\|}}{1+i \pi A_{0}^{\|}}\right|^{2} k_{B} L=\alpha Q^{2} \frac{1}{\tau^{2} k_{B}^{2} \Omega_{2}^{2}}\left|4 \pi \frac{A_{0}^{\|}}{1+i \pi A_{0}^{\|}}\right|^{2} k_{B} L .
$$

It is also convenient to write (63) in the form (the lattice is assumed to be square and have a period $d$ ):

$$
N_{p h}^{\|} \approx \alpha Q^{2} \frac{1}{8 \pi^{3} m^{2}} M_{\|}\left(\frac{\lambda_{B}}{d}\right)^{2} \frac{L}{\lambda_{B}} \approx 4 \cdot 10^{-5} Q^{2} \frac{1}{m^{2}} M_{\|}\left(\frac{\lambda_{B}}{d}\right)^{2} \frac{L}{\lambda_{B}},
$$

where $M_{\|}=\left|4 \pi \frac{A_{0}^{\|}}{1+i \pi A_{0}^{\|}}\right|^{2}$.

Let us analyze the number of quanta (63) in the frequency range from $100 \mathrm{GHz}$ to $3 \mathrm{THz}$. It will be recalled that the expressions for the coefficients $\frac{A_{0}^{\|}}{1+i \pi A_{0}^{\|}}$, derived in [12], are valid for the the case when $k R \ll 1$ ( $R$ is the radius of the thread), and so all the following conclusions are valid for the case $k R \ll 1$.

However, for $k R \geq 1$, the proposed analysis cannot be applied, and a more complicated theory is required.

The number of quanta (64) includes the factor $M_{\|}=\left|4 \pi \frac{A_{0}^{\|}}{1+i \pi A_{0}^{\|}}\right|^{2}$, which determines the behavior of the number of the emitted quanta as a function of the radiation frequency, the type and the radius of threads. The denominator of $M_{\|}$has the form $\pi^{2} \operatorname{Re} A 0^{2}+(1-\pi \operatorname{Im} A 0)^{2}$ and for perfectly conducting 


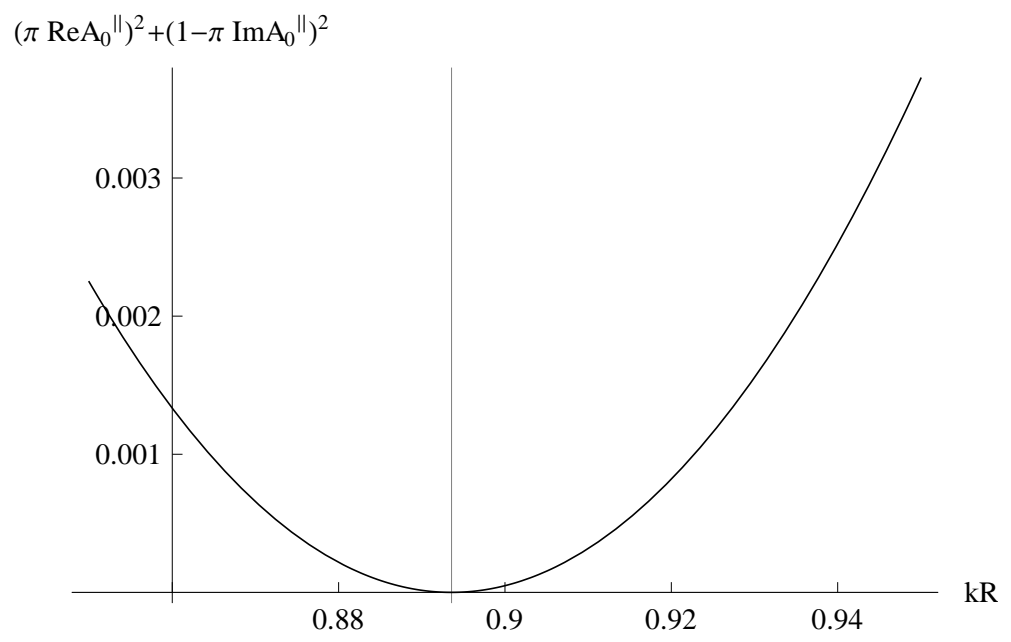

Figure 2: Denominator of $M_{\|}$for perfectly conducting threads as a function of $k R$

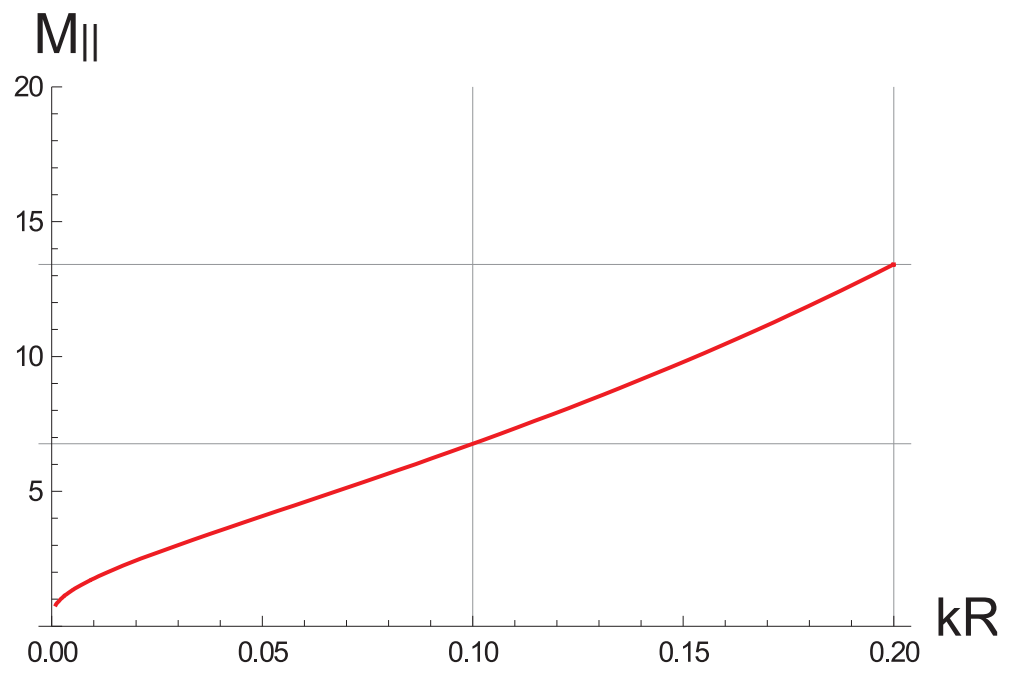

Figure 3: Factor $M_{\|}$for perfectly conducting threads as a function of $k R$

threads appears to be zero at $k R \approx 0.89$ (see Fig.2 and equation $(10)$ in [12]).

So, close to $k R \approx 0.89$, the factor $M_{\|}$and therefore the number of quanta exhibit a sharp growth.

As follows from Fig, 3 , in the range $k R \approx 0.2$, the factor $M_{\|}$is of the order of ten. As a result, (64) yields the following estimate for the number of quasi-Cherenkov radiation quanta having a frequency of about $1 \mathrm{THz}$ and emitted by an electron over $1 \mathrm{~cm}$ path length (it is assumed that $m=1$ and $d \sim 0.3 \mathrm{~cm})$

$$
N_{p h}^{\|} \approx 10^{-5} M_{\|}
$$

for example, for $M_{\|}=10$ over the length $L=10 \mathrm{~cm}$, we have

$$
N_{p h}^{\|}=10^{-3} \text { quanta. }
$$

Simple estimations can give the number of radiated quanta for an electron bunch passing through (or moving along the surface) of a photonic crystal [8, 45]. Suppose the bunch duration is $\tau_{b}=10^{-12} \mathrm{~s}$ (i.e., the length of about the radiation wavelength) and the number of electrons is $n_{e}=10^{9}$ [46, 47]. 
Then the radiation power, considering quanta with polarization parallel to the thread's axis, is as follows

$$
P=\frac{N_{p h}^{\|} n_{e}^{2} \hbar \omega}{\tau_{b}} \approx 2 \pi 10^{5} \mathrm{~W},
$$

and for bunches with the number of electrons $n_{e} \sim 10^{11}$ the power approaches a gigawatt level. In this case, particle bunches obtained at acceleration with ultra-intense and ultrashort laser pulses are promising for the creation of a trehertz radiation source with significant power.

Note that (64) includes the dependance on the uncompensated ion charge $Q$ as $Q^{2}$, therefore for $Q \sim 30$ we can expect the radiation yield as high as a quantum per particle under the same conditions as in (65). Thus, photonic crystals can be used to detect high energy charged particles; moreover, the particle beam need not necessarily pass through the crystal: it can move along the crystal surface at a distance $\delta<\frac{\lambda \beta \gamma}{4 \pi}$, which, for example, for $\lambda \sim 1 \mathrm{~mm}$ and particles with the energy $50 \mathrm{MeV}(\gamma=100)$ is as large as $\delta \sim 8 \mathrm{~mm}$.

In conditions of surface diffraction photonic crystals could be applied for high-energy particle detection by emitting quanta at a large angle to the particle momentum. Let us note that even in the optical range, e.g., for $\lambda \sim 10^{-4} \mathrm{~cm}$ and $\gamma=10^{6}$, the distance $d \sim 10 \mathrm{~cm}$.

The above-described increase in the number of quanta emitted by a particle in the range close to $k R \sim 1$ also occurs in the case of orthogonal polarization. Taking account of a final conductivity of metals has little influence on the obtained estimate of the number of quanta. According to [42], for this polarization an ordinary Cherenkov effect is possible. As follows from the above consideration, in the range close to $k R \sim 1$, the number of Cherenkov radiation quanta also increases. A similar increase in the number of quanta is also observed in the case of diffraction radiation, Smith-Purcell effect, and surface quasi-Cherenkov radiation, at which particles move along the surface of such photonic crystals.

\section{Conclusion}

The expressions for spectral-angular distribution of quasi-Cherenkov radiation emitted by a relativistic particle traversing a photonic crystal are derived. It is shown that for a relativistic particle passing through a photonic crystal formed by periodically strained threads, the intensity of Cherenkov radiation emitted at small angles to the direction of particle motion, as contrasted to ordinary Cherenkov radiation, exhibits anisotropic properties as the photon momentum is rotated about the direction of particle motion (as the crystal is rotated about the direction of particle motion at fixed-angle observation of the outcoming photon).

The intensity of quasi-Cherenkov radiation in terahertz and optical ranges is shown to be high enough to allow the experimental study of quasi-Cherenkov radiation.

Some applications of the described phenomena are considered:

In conditions of surface diffraction photonic crystals could be applied for high-energy particle detection by emitting a quanta at a large angle to the particle momentum. 
When passing through a photonic crystal, the particle bunches obtained at acceleration with ultra-intense and ultrashort laser pulses are promising for the creation of a terahertz radiation source with significant power.

\section{Acknowledgement}

Because there is a thermal background in this range, $\hbar \omega=k T$, the induced radiation becomes important. We want to thank Sergei Anishchenko for pointing out that this fact should be taken into account [48 50].

\section{References}

[1] R. Kompfner, Wireless World 52, 369 (1946).

[2] R. Pierce, Proc. IRE 35, 111 (1947).

[3] S.J. Smith and E.M. Purcell, Phys. Rev. 92, 1069 (1953).

[4] B. M. Bolotovskii and G. V. Voskresenskii, Usp. Fiz. Nauk. 88, 209 (1966) (Sov. Phys. Usp. 9, 73 (1966)).

[5] M.L. Ter-Mikaelian. The Influence of the Medium on High Energy Processes at High Energies, Publishing House of Acamemy of Science of Armenia, Yerevan, 1969 [In Rissian]; High Energy Electromagnetic Processes in Condensed Media New York, Wiley, 1972.

[6] V.G. Baryshevsky, Scattering of light by a flow of electrons through a crystal, Dokl. Akad. Nauk BSSR 15, 306 (1971).

[7] V.G. Baryshevsky, I.D. Feranchuk, A.P. Ulyanenkov, Parametric XRay Radiation in Crystals: Theory, Experiment and Applications, Series: Springer Tracts in Modern Physics, Vol. 213, 2005.

[8] V.G. Baryshevsky, High-Energy Nuclear Optics of Polarized Particles, World Scientific Publishing, Singapore, 2012.

[9] Baryshevskii, V. G. and Feranchuk, I. D. Transition radiation of $\gamma$ rays in a crystal, Zh. Eksp. Teor. Fiz. 61, 944 (1972); (Sov. Phys. JETP 34, 502 (1972).

[10] A.N. Didenko, B.N. Kalinin, S. Pak, A.P. Potylitsin, S.A. Vorobiev, V.G. Baryshevsky, V.A. Danilov, I.D. Feranchuk, Observation of monochromatic X-ray radiation from $900 \mathrm{MeV}$ electrons transmitting through a diamond crystal, Phys. Lett. 110A, N 3, 177 (1985).

[11] Yu.N. Adishchev, V.G.Baryshevskii, S.A. Vorob'ev, V.A. Danilov, S.D. Pak, A.P. Potylitsyn, P.F. Safronov, I.D. Feranchuk, S.V. Cherepitsa, Experimental observation of parametric x-ray emission, JETP Lett. 41, n.7, 361 (1985).

[12] V.G. Baryshevsky, A.A. Gurinovich, Spontaneous and induced parametric and Smith-Purcell radiation from electrons moving in a photonic crystal built from the metallic threads, Nuclear Instrum and Methods 252B 92 (2006). 
[13] Baryshevsky, V.G., Batrakov, K.G., Dubovskaya, I.Ya., Karpovich, V.A. and Rodionova, V.M., Volume quasi-Cherenkov FEL in mmspectral range, in G. Dattoli and A. Renieri (eds.) Free Electron Lasers pp. II-75-II-76, Elsiver Science B.V. (1997).

[14] X. Artru, P. Rullhunsen, Parametric X-rays and diffracted transition radiation in perfect and mosaic crystals, Nuclear Instrum. and Methods 173B16 (2001).

[15] P. P. Ewald, Fifty Years of X-Ray Diffraction (Reprinted in pdf format for the IUCr XVIII Congress, Glasgow, Scotland, Copyright (c) 1962, 1999 International Union of Crystallography) http://www.iucr.org/publ/50yearsofxraydiffraction

[16] R. W. James, The Optical Principles of the Diffraction of X-rays, Ox Bow Press, 1982.

[17] Z. G. Pinsker, Dynamical Scattering of X-Rays in Crystals, Springer, Berlin, 1978.

[18] Chang Shih-Lin, Multiple Diffraction of X-Rays in Crystals, SpringerVerlag Berlin Heidelberg New York Tokyo, 1984.

[19] L. D. Landau, E. M. Lifshitz, and L. P. Pitaevskii, Electrodynamics of Continuous Media: in L. D. Landau, and E. M. Lifshitz, Course of Theoretical Physics Vol. 8, 2nd edn., Butterworth-Heinemann, 1984.

[20] S. V. Anishchenko, V. G. Baryshevsky, A.A. Gurinovich, Time dependence of the intensity of parametric quasi-Cherenkov radiation produced by relativistic particles passing through electromagnetic (photonic) crystals, J. Nanophoton. 6 (1), 061714-1-061714-13 (2012).

[21] S. V. Anishchenko, V. G. Baryshevsky, Parametric (quasi-Cherenkov) cooperative radiation produced by electron bunches in natural or photonic crystals, LANL e-print arXiv:1304.0960

[22] V. G. Baryshevsky, Parametric X-ray radiation at a small angle near the velocity direction of the relativistic particle, Nucl. Instrum. Methods 122B, 1, 13 (1997).

[23] P.M. Morse, H. Feshbach, Methods of Theoretical Physics, Mc Graw Hill, New York, 1953.

[24] W. Friedrich, P. Knipping and M. Laue Interferenzerscheinungen bei Röntgenstrahlen, Ann. Phys. 41971 (1913).

[25] B.W. Batterman, H. Cole, Dynamical diffraction of $\mathrm{X}$ rays by perfect crystals, Rev. Mod. Phys. 36, n.3, 681 (1964).

[26] Sommerfeld, A. and H. Bethe, Electron Theory of Metals, Handbuch der Physik 24, 2, (1933).

[27] V. G. Baryshevsky, Surface parametric radiation of relativistic particles, Dokl. Akad. Nauk SSSR 299, 6, 1363 (1988). 
[28] E. Yablonovitch, Inhibited spontaneous emission in solid-state physics and electronics, Phys. Rev. Lett. 58, 2059 (1987).

[29] E. Yablonovitch, Photonic Crystals: Semiconductors of Light, Scientific American, 285, no. 6, 47 (2001).

[30] E. I. Smirnova, C. Chen, M. A. Shapiro, J. R. Sirigiri, and R. J. Temkin, Simulation of photonic band gaps in metal rod lattices for microwave applications, J. Appl. Phys. 91, 3, 960 (2002).

[31] E.I. Smirnova, I. Mastovsky, M.A. Shapiro, R.J. Temkin, L.M. Earley and R.L. Edwards, Fabrication and cold test of photonic band gap resonators and accelerator structures, Phys. Rev. Special Topics: Accelerators and Beams 8, 091302 (2005).

[32] A. L. Pokrovsky, Analytical and numerical studies of wire-mesh metallic photonic crystals, Phys. Rev. 69B, 195108 (2004).

[33] J. R. Sirigiri, K. E. Kreischer, J. Machuzak, I. Mastovsky, M. A. Shapiro, and R. J. Temkin, Photonic-band-gap resonator gyrotron, Phys. Rev. Lett. 86, 5628 (2001).

[34] M. A. Shapiro, W. J. Brown, I. Mastovsky, J. R. Sirigiri, and R. J. Temkin, $17 \mathrm{GHz}$ photonic band gap cavity with improved input coupling, Phys. Rev Special Topics: Accelerators and Beams 4, 042001 (2001).

[35] S. Ya. Kilin and D. S. Mogilevtsev, Quantum Optics Methods of Structured Reservoirs, Minsk, Belorusskaya Nauka, 2007 [in Russian].

[36] S. Kilin, D. Mogilevtsev, Two-dimentional photonic crystals, Physics of Low Dimensional Structures, Vol. 1. p. 207 (1999).

[37] M.G. Silveirinha, Nonlocal homogenization model for a periodic array of epsilon-negative rods, Phys.Rev. 73E, n.4, 046612 (2006).

[38] E. I. Smirnova, C. Chen, M. A. Shapiro and R. J. Temkin, Simulation of Photonic Band Gaps in Metal Rod Lattices for Microwave Applications, J. Appl.Phys. 91, n.3, 960 (2002).

[39] T. Suzuki, P.K.L. Yu, Method of projection operators for photonic band structures with perfectly conducting elements, Phys. Rev. 57B, n.4, 2229 (1998)

[40] N. A. Nicorovici, R. C. McPhedran, and L. C. Botten, Photonic band gap for arrays of perfectly conducting cylinders, Phys. Rev. 52E, 1135 (1995).

[41] V.V. Nikolsky, Electrodynamics and Propagation of Radio-Wave, Nauka, 1978.

[42] V.G. Baryshevsky, E.A. Gurnevich. Dynamical diffraction theory of waves in photonic crystals built from anisotropically scattering elements, Journal of Nanophotonics 6 n.1, 061713 (2012). 
[43] S.A. Maksimenko, Effect of slab interfaces on diffraction of visible light by a thick volume grating, Journal of Modern Optics 41, n.10, 1875 (1994).

[44] V.G. Baryshevsky, Channeling, Radiation and Reactions in Crystals at High Energy, Bel. State Univers., Minsk, 1982.

[45] S.V. Anishchenko, V.G.Baryshevsky, Parametric (quasi-Cherenkov) cooperative radiation produced by electron bunches in natural or photonic crystals, LANL e-print arXiv:1304.0960v1.

[46] K. Ta Phuoc, S. Corde, C. Thaury, V. Malka, A. Tafzi, J. P. Goddet, R. C. Shah, S. Sebban and A. Rousse, All-optical Compton gamma-ray source, Nature Photonics 6, 308 (2012) (doi:10.1038/nphoton.2012.82).

[47] O. Lundh, J. Lim, C. Rechatin, L. Ammoura, A. Ben-Ismaïl, X. Davoine, G. Gallot, J-P. Goddet, E. Lefebvre, V. Malka and J. Faure, Few femtosecond, few kiloampere electron bunch produced by a laser-plasma accelerator, Nature Physics 7, 219 (2011) (10.1038/nphys1872).

[48] Ginzburg V.L., Some questions of the theory of electric fluctuations, Usp. Fiz. Nauk 46, issue 3, 348 (1952).

[49] Fain V.M., Quantum phenomena in radio frequency range, Usp. Fiz. Nauk 64, issue 2, 273 (1958).

[50] S.V. Anishchenko, Superradiance startup at finite temperatures of the electromagnetic field, LANL e-print arXiv:14061483v1 [physics.optics]. 